\title{
Aplicação do Laser na Cariologia: diagnóstico, prevenção e tratamento
}

Application of laser in cariology: diagnosis, prevention and treatment Aplicación del láser en la cariología: diagnóstico, prevención y tratamiento

\author{
Ana Luzia Araújo BATISTA ${ }^{1}$ \\ Thamyres Maria Silva SIMÕES ${ }^{1}$ \\ Maria das Graças Barbosa da SILVA ${ }^{\mathbf{1}}$ \\ Maria Helena Chaves de Vasconcelos CATÃo ${ }^{2}$

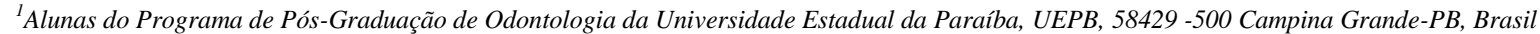 \\ ${ }^{2}$ Professora do Programa de Pós-Graduação de Odontologia da Universidade Estadual da Paraíba, UEPB, 58429 -500 Campina Grande-PB, Brasil
}

\section{Resumo}

Nas últimas décadas, um dos grandes avanços na Área da Saúde foi o desenvolvimento dos aparelhos de Laser, que possibilitou uma nova abordagem nos procedimentos odontológicos. A Laserterapia tem diversas aplicabilidades na Cariologia, seja como método de diagnóstico, como auxiliar nas medidas preventivas ou como tratamento da cárie dentária. Portanto, o objetivo desta revisão é proporcionar uma leitura atualizada da aplicabilidade da Laserterapia na Cariologia e, para tanto, foi realizada uma revisão literária sistematizada, na base de dados MEDLINE - Pubmed, abrangendo os últimos anos. Mediante esse estudo literário e considerando a evolução dos estudos científicos, ainda existem controvérsias a respeito da eficácia clínica do Laser na Cariologia.

Descritores: Lasers; Cárie Dentária; Flúor.

\section{Abstract}

In the last decades, one of the great advances in the Health Area was the development of the Laser devices, which made possible a new approach in dental procedures. Laser therapy has several applicability in Cariology, either as a diagnostic method, as an aid in preventive measures or as a treatment of dental caries. Therefore, the purpose of this review is to provide an updated reading of the applicability of laser therapy in Cariology and, for this purpose, a systematized literary research was carried out in the MEDLINE - Pubmed database, covering the last years. Through this literary study and considering the evolution of scientific studies, there are still controversies regarding its clinical efficacy of the laser in Cariology.

Descriptors: Lasers; Dental Caries; Fluorine.

\section{Resumen}

En las últimas décadas, uno de los grandes avances en el área de la salud fue el desarrollo de los aparatos láser, que posibilitó un nuevo abordaje en los procedimientos odontológicos. La Laserterapia tiene diversas aplicabilidad en la Cariología, sea como método de diagnóstico, como auxiliar en las medidas preventivas o como tratamiento de la caries dental. Por lo tanto, el objetivo de esta revisión es proporcionar una lectura actualizada de la aplicabilidad de la láserterapia en la Cariología y, para ello, se realizó una investigación literaria sistematizada, en la base de datos MEDLINE - Pubmed, abarcando los últimos años. Mediante este estudio literario y considerando la evolución de los estudios científicos, todavía existen controversias acerca de su eficacia clínica del láser en la Cariología.

Descriptores: Rayos Láser; Caries Dental; Flúor.

\section{INTRODUÇÃO}

A cárie dentária $(\mathrm{CD})$ é um dos problemas mais importantes na Área da Saúde Mundial por ser a doença mais comum na infância e idade adulta e a principal causa de perda dentária ${ }^{1}$.

A prevalência é bastante elevada, atingindo quase $100 \%$ da população $0^{2,3}$, embora os números de casos incidentes tenham diminuído ${ }^{4}$, em virtude de uma melhoria nas condições de higiene oral ${ }^{5}$.

Por ser considerado um problema de Saúde Pública, é importante ter um diagnóstico precoce e preciso $^{1}$, quando o tratamento não-invasivo, com abordagens preventivas ainda é viável, preservando estrutura dental ${ }^{6}$, resultando em menor custo e uma manutenção por mais tempo do dente na cavidade bucal $^{5}$.

O grande desafio é identificar as lesões nos estágios iniciais ${ }^{6,7}$, cárie em sulcos e fissuras ${ }^{5}$, cárie oclusal não-cavitada ${ }^{6}$ e cárie sob restauração ${ }^{1,8}$ por serem de difíceis diagnóstico.

Além dos métodos convencionais mais utilizados na prática clínica, tais como o exame tátil, visual e radiográfico ${ }^{9}$, uma série de novos sistemas de diagnóstico, utilizando propriedades fluorescente do Laser, têm sido desenvolvidos ${ }^{1,5,6}$.

O Laser também tem sido usado na prevenção da cárie dentária ${ }^{10}$, juntamente com a aplicação de flúor, aumentando a sua absorção e servindo como reservatório durante o processo remineralização ${ }^{4}$.
A aplicação do Laser no tratamento da cárie pode ser por intermédio da Terapia Fotodinâmica (TFD), com o intuito de eliminar as bactérias cariogênicas ${ }^{11}$ ou por atuação na remoção do tecido cariado e no preparo de cavidade ${ }^{12}$.

Portanto, o objetivo dessa revisão literária é proporcionar uma leitura atualizada da aplicabilidade da Laserterapia na Cariologia, ressaltando as indicações e controvérsias, fornecendo conhecimentos atualizados para aplicação segura na Clínica Odontológica.

REVISÃO DA LITERATURA

- Laser na Odontologia

O Laser consiste num dispositivo que contém uma cavidade óptica altamente reflexiva constituído por um material com propriedade de permitir a amplificação da luz por emissão estimulada. A luz do Laser é uma onda eletromagnética com emissão estimulada, extremamente direcionada e com baixa divergência no espaço ${ }^{2}$.

Os Lasers podem ser encontrados em diferentes estados como gás (gás carbônico, argônio), líquido (corante orgânico), sólidos (Nd:YAG) ou plasma (semicondutores). Para explorar as interações entre o Laser e os tecidos odontológicos, diferentes tipos de Lasers têm sido operado, variando uma série de parâmetros ${ }^{2}$. 
Os Lasers podem ser classificados em Laser de baixa intensidade e Laser de alta intensidade. $\mathrm{O}$ Laser de baixa intensidade, por meio das reações fotoquímicas, pode ser aplicado no diagnóstico e na prevenção da cárie dentária, na desinfecção de canais radiculares, no tratamento de bolsas periodontais e da periimplantite e no clareamento dental ${ }^{12}$. Eles são relativamente barato, de pequeno porte, fácil de transportar e têm múltiplas aplicações em várias áreas da Odontologia ${ }^{10}$

O Laser de alta intensidade, por sua vez, pode ser usado no tratamento de doenças malignas, na remoção de tecido cariado, no preparo de cavidades $^{12}$, na cirurgia de tecidos moles e para melhorar a resistência da estrutura do dente à desmineralização ${ }^{13}$.

A irradiação do Laser no tecido dental leva a uma variedade de alterações estruturais e essas mudanças dependem de parâmetros de irradiação, tais como, comprimento de onda, modo de emissão de Laser, densidade de energia, duração de pulso, taxa de repetição e propriedades de tecido-alvo ${ }^{12}$.

A interação do Laser com os tecidos dentais (esmalte e dentina) são classificados em três categorias principais: interação com o mineral (hidroxiapatita carbonatada), interação com a água e interação com material orgânico (proteínas e lipídeos). A dentina tem maior teor de água e de proteína em compração com o esmalte, propiciando a absorção de luz e ao mesmo tempo permitindo que ela se espalhe, podendo levar a consequências negativas, tais como vaporização, rachaduras e necrose pulpar ${ }^{2}$.

\section{- Cárie dentária}

A cárie dentária apresenta como fator etiológico a bactéria patogênica específica (Streptococus mutans) que metaboliza carboidratos para a formação de ácidos ${ }^{3}$, ou seja, é uma doença biofilme-dependente ${ }^{14}$. Tal condição é resultado do desequilíbrio entre fatores patológicos que levam à desmineralização e de fatores de proteção que aumentam a resistência aos ácidos do esmalte ou promovem a sua capacidade remineralizante ou ambos $^{2}$.

Dentre os fatores patológicos pode-se incluir a higiene oral inadequada, a dieta com a ingestão excessiva de açúcar (quantidade e frequência) e a redução dos mecanismos naturais (fluxo salivar, mastigação e motilidade da língua). Entre os fatores de proteção, tem-se o fluxo salivar, os componentes salivares, os antibacterianos (natural e aplicados), o fluoreto de fontes extrínsecas e os componentes alimentares selecionados ${ }^{2}$.

Fatores nutricionais também tem papel significante pelo potencial erosivo de diferentes substâncias. Doenças sistêmicas como refluxo gastroesofágico e estrutura do esmalte com predisposição anatômica também são considerados fatores de risco por deixar a estrutura dental mais fragilizada e susceptível ao desenvolvimento da cárie dentária $^{12}$.

A descalcificação do esmalte ou a formação de manchas brancas é o primeiro sinal de cárie. Se o tratamento adequado é apresentado para lesões em esmalte, é possível inverter a condição através do processo de remineralização, envolvendo a difusão de minerais para a estrutura do dente ${ }^{10}$. No entanto, se não tratada, pode provocar danos progressivos aos tecidos dentais que vai desde manchas brancas, até cavitações mais profundas, com envolvimento pulpar, deixando os dentes fragilizados e com sua função comprometida, levando até mesmo a sua perda ${ }^{2}$.

- Diagnóstico da Cárie Dentária

Com o progresso da tecnologia e o surgimento de equipamentos de detecção da cárie dentária, tornou-se possível realizar o diagnóstico nos estágios iniciais, resultando em menos custos no tratamento, uma maior permanência de tempo do dente na cavidade oral ${ }^{5}$, tratamento minimamente invasivo, por meio de abordagens mais preventivas com agentes remineralizantes e selagem e preservação da estrutura do dente ${ }^{6}$.

O grande desafio é a detecção de cárie em sulcos e fissuras ${ }^{5}$, cárie oclusal não-cavitada e cárie sob restaurações ${ }^{8}$. Vários métodos estão disponíveis, sendo os mais utilizados o visual, o tátil e o radiográfico ${ }^{9}$. Entretanto, uma série de novos sistemas de diagnóstico têm sido desenvolvidos, utilizando as propriedades fluorescente dos tecidos duros do dente, baseando-se no princípio de que os tecidos cariados alteram as propriedades de fluorescência em comparação com o tecidos dentais sadios. Pode-se citar: a fluorescência induzida por luz quantitativa (FLQ), a fluorescência a Laser (LF ou DIAGNOdent) e o VistaProof (luz de diodo

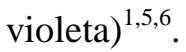

O método de fluorescência induzida por luz quantitativa (FLQ) usa uma lâmpada de halógeno que emite uma luz azul com um comprimento de onda de 370nm que excita a estrutura do dente e, sem seguida, emite fluorescência. As imagens são então capturadas em um software que quantifica a perda de fluorescência provocada pela desmineralização, indicando perda mineral ${ }^{7}$.

O DIAGNOdent utiliza fluorescência a Laser (Laser diodo vermelho - $655 \mathrm{~nm}$ ) para medir diferenças de emissão de luz entre o tecido dental sadio e as lesões de cárie, uma vez que as porfirinas, produtos metabólicos microbianos, reemite parte da luz irradiada dentro do espectro infravermelho e pode ser quantificada em uma escala que vai de 0 a $99^{1,5-9,14}$.

Trata-se de um método quantitativo para o diagnóstico da cárie dentária e apresenta algumas vantagens, tais como, o fato de não ser invasivo; permitir a detecção precoce; possibilitar que a radiação penetre através do material restaurador para 
identificar a cárie; facilidade de uso; não necessita da cooperação do paciente; rápida determinação da condição de saúde bucal do paciente ${ }^{5}$; elevada repetibilidade $^{1,8,9}$; não produzir radiação ionizante nociva; capacidade de minimizar o erro humano ${ }^{1}$.

A sensibilidade do DIAGNOdent, que consiste na capacidade de um teste de diagnóstico para detectar corretamente a presença de cárie, varia de $44 \%$ a $100 \%$, enquanto que a especificidade, refere-se a capacidade de um teste para detectar corretamente a ausência de cárie, varia 36 a 100\% $(5,6)$. Ter uma alta sensibilidade faz com que este dispositivo apresente alta probabilidade de diagnóstico falso-positivo e, portanto, seu uso é recomendado em combinação com outras técnicas ${ }^{5}$.

Vale ressaltar que soluções antissépticas e componentes do material restaurador podem provocar alterações na estrutura dos dentes e afetar a resposta obtida a partir fluorescência ${ }^{5}$. Para minimizar os resultados falso-positivos, sugere-se o polimento da restauração ou superfície do dente com pedra-pomes antes das medições para eliminar as manchas e corrigir algumas limitações ${ }^{1,5,8}$.

O DIAGNOdent é um dispositivo que foi introduzido no mercado para detectar lesões oclusais e em superfícies lisas. No entanto, ele foi substituído por um cabo livre do tipo caneta de fluorescência a Laser (LF - pen) que, adicionalmente, permitiu a examinação das superfícies proximais, com o mesmo princípio de funcionamento ${ }^{7,9}$.

Mais recentemente, uma câmera de fluorescência VistaProof foi desenvolvida para detectar cárie oclusais. Essa método utiliza a luz de diodo (LED) violeta com um comprimento de onde de $405 \mathrm{~nm}$ para capturar a fluorescência vermelha da protoporfirina IX, um metabólito bacteriano que aparece na dentina cariada e no biofilme maduro, sendo quantificado de 0 a $5^{6,7}$. O tecido saudável, por sua vez, gera a fluorescência verde característica da dentina, proporcionando assim um "mapa" das áreas afetadas. A sensibilidade varia de 49 a $90 \%$ enquanto que a especificidade tem valores entre 63 e $80 \%$.

O que dificulta o diagnóstico preciso da cárie dentária é o fato de que essas lesões podem ocorrer numa estrutura altamente mineralizada, numa variedade de locais diferentes, com aspectos únicos de configuração e taxa de propagação. Todos esses aspectos tornam improvável que uma modalidade de diagnóstico terá sensibilidade e especificidade suficientes ${ }^{2}$. Portanto, concluir qual o método ideal pode ser muito difícil, considerando os variados sistemas, as diferentes categorizações, o tipo de dente, a superfície a ser avaliada, o nível de habilidade do operador e os diferentes métodos de análise ${ }^{5}$.

\section{- Prevenção da Cárie Dentária}

Os métodos para prevenção da cárie dentária incluem a aplicação tópica de flúor (ATF), uso de cremes dentais e enxaguatórios contendo flúor e a fluoretação de água potável ${ }^{4,5}$. Essas medidas preventivas têm diminuído a incidência dessa condição, uma vez que, tendem a aumentar o teor de minerais da saliva e, consequentemente, diminuir a desmineralização do esmalte ${ }^{4}$.

O mecanismo do Laser de baixa intensidade na prevenção da cárie pode estar relacionada aos seus efeitos fotoquímicos, uma vez que seu comprimento de onda, na faixa de 450 a $800 \mathrm{~nm}$ têm baixa absorção em esmalte ${ }^{10}$. A irradiação do Laser diodo, combinado com a aplicação de flúor, aumenta a absorção de fluoreto nos dentes permanentes e inibe danos causados por ataque ácido in vitro ${ }^{4,15}$.

O Laser de alta intensidade, por seus efeitos fototérmicos, é responsável pela modificação da estrutura de tecido duro dental, incluindo a redução do teor de carbonato e alterações na matéria orgânica, que conduz a um aumento na resistência ao ácido ${ }^{10,16}$. Tanto o Laser de $\mathrm{CO}_{2}$ como os de érbio (Er:YAG e Er, Cr:YSGG) são eficazes na prevenção de cáries, uma vez que são grandemente absorvido pela água e hidroxiapatita dos tecidos dentais e, portanto, capazes de modificar a estrutura cristalina $^{4,10,17}$.

A utilização do Laser juntamente com a aplicação de flúor tem ação por dois mecanismos: o aumento da penetração e absorção de fluoreto pelo esmalte, devido ao calor gerado pelo Laser e a retenção de flúor nas porosidades da superfície do esmalte e microfissuras ${ }^{4}$.

O calor gerado pela luz do Laser de alta intensidade leva a decomposição da matriz orgânica, a perda de água e de carbono, a formação das fases refratárias da hidroxiapatita (fosfato de cálcio ou pirofosfato de cálcio), converte fosfato ácido em pirofosfato carbonato, o que leva a perda de água do esmalte $^{4,12}$.

Os mecanismos pelos quais a irradiação do Laser aumenta a resistência do esmalte à cárie, envolvem a fusão de superfície e recristalização dos cristais de hidroxiapatita do esmalte, redução da permeabilidade e da solubilidade do esmalte e deposição de fluoreto de cálcio, aumentando a resistência do esmalte aos ácidos e assim desempenhando papel importante na prevenção de cáries $^{4,12,17}$.

A formação de fluorapatita e fluoreto de cálcio na superfície do esmalte servem como reservatório de fluoreto. Ademais, a maior absorção do flúor leva a maior dureza do esmalte, indicativo de conteúdo mineral, oferecendo maior resistência à desmineralização $0^{4,12,15,17}$. À medida que os cristais de esmalte perdem carbonato, tornam-se mais estáveis e mais resistentes a substâncias, conduzindo à obstrução de espaços interprismática e diminuição da permeabilidade $^{4,12}$.

\section{- Tratamento da Cárie Dentária}

A cárie dentária destrói progressivamente os tecidos do dente, de modo que seu tratamento inclui a 
remoção dos tecidos cariados. O sistema tradicional de remoção compreende o uso de brocas rotativas, sozinhas ou em combinação com instrumentos manuais, e tem um alto potencial de desencadear ansiedade e desconforto, decorrente do ruído e vibração mecânica da preparação do dente. Além do mais, a utilização de meios mecânicos pode levar a cavitação excessiva e resultar na perfuração da câmara pulpar ou, ao contrário, a remoção incompleta da dentina cariada ${ }^{2}$.

As desvantagens causadas pelo método tradicional levaram a uma busca por novas alternativas e estratégias no tratamento, para atingir uma conservação máxima de tecidos ${ }^{2}$. Portanto, nos últimos anos, tem havido um crescente interesse no uso de agentes químicos como antissépticos ou antibióticos como substituto ou complementar ${ }^{3,11}$.

Devido as limitações das técnicas anteriormente citadas, incluindo o desenvolvimento da resistência aos antibióticos, a utilização da tecnologia a Laser surgiu como uma abordagem alternativa. A Terapia Fotodinâmica antibacteriana foi introduzida como uma possibilidade de tratamento contra os fatores etiológicos da cárie e esse método consiste na utilização de um agente fotossensibilizador sendo ativado por uma luz com comprimento de onda específico, na presença de oxigênio $^{3,11}$.

Quanto aos fotossensibilizadores, tem sido usado o azul de toluidina, azul de metileno, rosa bengala, eritrosina, entre outros ${ }^{11}$. Com relação à luz empregada na TFD, pode-se utilizar o Laser, o LED, as lâmpadas de tungstênio e as halógenas ${ }^{3,11}$.

Os primeiros Lasers utilizados para ablação dental foram o Laser de rubi, Nd:YAG, Ho:YAG e $\mathrm{CO} 2$ que provocaram um aumento de temperatura na polpa, bem como microfissuras e carbonização. Entretanto, o uso do Laser de Er:YAG mostrou que os danos térmicos eram mínimos quando utiliza-se as configurações apropriadas e quando do emprego de um spray de arrefecimento de água ${ }^{2}$. Dependendo da duração do pulso, juntamente com a pulverização de ar/água, é possível evitar a formação de microfissuras e fendas no esmalte, assim como o aumento da temperatura na câmara pulpar ${ }^{12}$.

Para a remoção da cárie, o comprimento de onda do Laser deve ser de tal modo que haja uma interação tanto com o mineral tanto com a água. Por esse motivo, os Lasers de érbio, Er:YAG e Er:YSGG, são fortemente absorvidos pela água, o que facilita o principal mecanismo de ação que é o aquecimento da água na superfície ${ }^{2}$.

\section{CONCLUSÃO}

Mediante os conhecimentos expostos nessa revisão sistematizada da literatura, pode-se inferir que a laserterapia tem conseguido grandes avanços na Área Odontológica e na Cariologia tem aplicabilidade no diagnóstico, na prevenção e no tratamento da cárie dentária.

Devido às limitações dos métodos convencionais no diagnóstico das lesões iniciais, os estudos baseados em fluorescência apresentam resultados semelhantes entre si, sem diferenças significativas.

Por fim, os efeitos protetivos do Laser contra a cárie dental, diz respeito ao aumento da resistência do esmalte à desmineralização ácida, quando usado simultaneamente com a aplicação de flúor, por aumentar sua incorporação ao esmalte.

\section{REFERÊNCIAS}

1. Sichani AV, Javadinejad S, Ghafari R. Diagnostic value of DIAGNOdent in detecting caries under composite restorations of primary molars. Dent Res J. 2016;13(4):327-32.

2. Montedori A, Abraha I, Orso M, D'errico PG, Pagano S, Lombardo G. Lasers for caries removal in deciduous and permanent teeth. Cochrane Database Syst Rev. 2016;26(9):CD010229.

3. Nagata JY, Hioka N, Kimura E, Batistela VR, Terada RS, Graciano AX et al. Antibacterial photodynamic therapy for dental caries: evaluation of the photosensitizers used and light source properties. Photodiagnosis Photodyn Ther. 2012;9(2):122-31.

4. Bahrololoomi Z, Fotuhi Ardakani F, Sorouri M. In vitro comparison of the effects of diode laser and $\mathrm{CO}_{2}$ laser on topical fluoride uptake in primary teeth. J Dent (Tehran). 2015;12(8):585-91.

5. Nokhbatolfoghahaie H, Alikhasi M, Chiniforush N, Khoei F, Safavi N, Yaghoub Zadeh B. Evaluation of Accuracy of DIAGNOdent in Diagnosis of Primary and Secondary Caries in Comparison to Conventional Methods. J Lasers Med Sci. 2013; 4(4):159-67.

6. Melo M, Pascual A, Camps I, Del Campo Á, AtaAli J. Caries diagnosis using light fluorescence devices in comparison with traditional visual and tactile evaluation: a prospective study in 152 patients. Odontology. 2017;105(3):283-90.

7. Gimenez T, Braga MM, Raggio DP, Deery C, Ricketts DN, Mendes FM. Fluorescence-based methods for detecting caries lesions: systematic review, meta-analysis and sources of heterogeneity. PLoS One. 2013;8(4):e60421.

8. Kositbowornchai S, Sukanya C, Tidarat T, Chanoggarn T. Caries detection under composite restorations by laser fluorescence and digital radiography. Clin Oral Investig. 2013; 17(9):2079-84.

9. Rosa MI, Schambeck VS, Dondossola ER, Alexandre MC, Tuon L, Grande AJ, Hugo F. Laser fluorescence of caries detection in permanent teeth in vitro: a systematic review and meta-analysis. J Evid Based Med. 2016;9(4):213-24. 
10.Heravi F, Ahrari F, Mahdavi M, Basafa S. Comparative evaluation of the effect of Er:YAG laser and low level laser irradiation combined with CPP-ACPF cream on treatment of enamel caries. J Clin Exp Dent. 2014;6(2):e121-26.

11.Mang TS, Tayal DP, Baier R. Photodynamic therapy as an alternative treatment for disinfection of bacteria in oral biofilms. Lasers Surg Med. 2012;44(7):588-96.

12. Fornaini C, Brulat N, Milia G, Rockl A, Rocca JP. The use of sub-ablative Er:YAG laser irradiation in prevention of dental caries during orthodontic treatment. Laser Ther. 2014;23(3):173-81.

13.Karandish M. The efficiency of laser application on the enamel surface: a systematic review. J Lasers Med Sci. 2014;5(3):108-14.

14. Twetman S, Axelsson S, Dahlén G, Espelid I, Mejàre I, Norlund A et al. Adjunct methods for caries detection: a systematic review of literature. Acta Odontol Scand. 2013;71(3-4):388-97.

15.Bahrololoomi Z, Lotfian M. Effect of diode laser irradiation combined with topical fluoride on enamel microhardness of primary teeth. J Dent (Tehran). 2015;12(2):85-9.

16. Ramos-Oliveira TM, Ramos TM, EstevesOliveira M, Apel C, Fischer H, Eduardo CP et al. Potential of $\mathrm{CO} 2$ lasers $(10.6 \mu \mathrm{m})$ associated with fluorides in inhibiting human enamel erosion. Braz Oral Res. 2014;28(1):1-6.

17. Souza-Gabriel AE, Turssi CP, Colucci V, Tenuta LM, Serra MC, Corona SA. In situ study of the anticariogenic potential of fluoride varnish combined with $\mathrm{CO} 2$ laser on enamel. Arch Oral Biol. 2015;60(6):804-10.
CONFLITO DE INTERESSES

Os autores declaram não haver conflitos de interesse.

AUTOR PARA CORRESPONDÊNCIA

Maria Helena Chaves de Vasconcelos Catão

mhelenact@zipmail.com.br

Submetido em 14/08/2018

Aceito em 04/10/2018 